\title{
Monitoring oxygen in the newborn: saturation or partial pressure?
}

Pulse oximeters are a new type of medical equipment. They have been welcomed warmly into adult intensive care and anaesthetics, where there was little rival technology and no fear of arterial hyperoxia. In neonatal intensive care, though, where efficient monitoring of partial pressure is well established and too much oxygen is almost as worrying as too little, the place of oximetry is less clear. The several oximeters now available are undeniably sophisticated instruments, but they are too expensive to be left gathering dust in the equipment cupboard because they failed to fulfil expectations. This article assesses what can and cannot be expected of oximetry in monitoring oxygen treatment in the newborn.

\section{How pulse oximeters work}

Oximeters make use of the fact that oxyhaemogloin and deoxyhaemoglobin absorb light at the red end of the spectrum differently; deoxyhaemoglobin absorbs more red than infrared and oxyhaemoglobin more infrared than red. The oximeter probe consists of a light emitter and a light sensor, which are aligned on opposite sides of a narrow part of the body, such as the palm or forefoot in the case of a baby. The emitter sends equal intensities of red and infrared light into the tissue, and the sensor detects the ratio of red to infrared that emerges. From this information the proportion of oxyhaemoglobin to deoxyhaemoglobin-that is, the percentage saturation of haemoglobin with oxygen-is calculated and displayed. As the instrument measures a ratio rather than an absolute value no external calibration is necessary.

So that the oximeter measures the saturation of arterial blood rather than capillary or venous blood the instrument is programmed to look only at pulsatile increases in oxyhaemoglobin concentration-hence the term 'pulse' oximetry. For accurate measurements of arterial saturation a reasonable pulse must be detected. Most available oximeters use a 'bouncing light' display to indicate the pulse amplitude and will give warning when the pulse is inadequate for reliable measurements. At least one model displays an image of the pulse waveform, which allows the user to judge the signal quality.

\section{Relation between saturation and partial pressure}

Normally, $98 \%$ of the oxygen in arterial blood is bound to haemoglobin and $2 \%$ is dissolved in plasma. Oxygen electrodes, whether in a blood gas analyser, an arterial catheter, or a transcutaneous monitor, measure the partial pressure of dissolved oxygen. In the physiological range, however, the amount of oxygen taken up by haemoglobin is a function of the partial pressure of dissolved oxygen, so that a measurement of partial pressure provides an assessment of the oxygen content of haemoglobin. The precise relation is described by the haemoglobin/oxygen dissociation curve, in which the oxygen content of haemoglobin is usually expressed as a percentage of the maximum possible content-that is, percentage saturation. The sigmoid shape of the curve, which is a key factor in any discussion of oximetry, is highly relevant to the function of haemoglobin. The flat section, which is principally concerned with oxygen uptake, ensures that arterial blood leaves the lung well saturated even when the partial pressure is reduced to about $9 \mathrm{kPa}$ - say by modest lung disease or living at altitude. The steep lower portion ensures an adequate oxygen supply to tissues by releasing large amounts of oxygen for relatively small decreases in partial pressure.

There are several important influences on the relation between partial pressure and saturation. Those most relevant to neonatology are the change from fetal haemoglobin to adult haemoglobin, either naturally or due to transfusion, and changes in $\mathrm{pH}$. Increasing concentrations of adult haemoglobin move the dissociation curve to the right, as does a fall in $\mathrm{pH}$. Both of these effects are most pronounced on the steep portion of the curve, and I discuss their influence on tissue oxygenation and the interpretation of oxygen tension and saturation below.

Most paediatricians are familiar with partial pressure and find saturation less comprehensible. Developing a feel for the relation between the two is helped by a ready means of conversion. Any programmable calculator can be used to convert saturation values to partial pressures and vice versa, making allowance for the type of haemoglobin and 
the $\mathrm{pH}$. Hellegers et al give the formulae needed for these calculations. ${ }^{1}$

\section{Tissue oxygenation}

The supply of oxygen to the tissues does not depend solely on the oxygenation of haemoglobin because blood flow, haemoglobin concentration, and tissue oxygen consumption are also important. In so far as it does depend on haemoglobin oxygenation, however, the important factor is the amount of oxygen released by haemoglobin as the partial pressure of oxygen in arterial blood equilibrates with the partial pressure of oxygen in the tissue. This is determined by the difference in the saturation of haemoglobin at the two partial pressures and is a property of the slope of the dissociation curve rather than of either the absolute partial pressure of oxygen in plasma or the absolute percentage saturation of haemoglobin. For example, the reason that fetal haemoglobin suits the fetus is that, between the partial pressure of oxygen in fetal blood (about 4 $\mathrm{kPa}$ ) and the partial pressure of oxygen in fetal tissue (about $2 \mathrm{kPA}$ ), the dissociation curve of fetal haemoglobin is steeper than that of adult haemoglobin. The fact that fetal haemoglobin is 'left shifted' compared with adult haemoglobin is important only in so far as it makes that dissociation curve steeper over the relevant range. The fact that fetal haemoglobin has a 'greater affinity for oxygen'-that is, a higher percentage saturation at a given partial pressure-is in itself irrelevant as far as tissue oxygenation is concerned; after all, carboxyhaemoglobin has a high affinity for oxygen. The purpose of this line of argument is to try to remove from the saturation/partial pressure debate the notion that either one alone is more relevant than the other to considerations of tissue oxygenation. Knowing both is quite a different matter.

\section{Interpreting measurements of partial pressure $\left(\mathrm{PaO}_{2}\right)$ and saturation $\left(\mathrm{SaO}_{2}\right)$}

Several published articles, including two in this issue, ${ }^{2}{ }^{3}$ have related oximeter readings to simultaneous measurements of partial pressure. They have all found a satisfactory overall correlation between the two, which describes the familiar shape of the oxygen dissociation curve of haemoglobin. A closer look, though, shows that over the upper section of the curve a wide range of partial pressure corresponds to a narrow range of saturation, and that over the lower portion of the curve the opposite is true. This effect is magnified in the published work because the graphs are constructed from pooled data from several babies; nevertheless, it is an inherent effect of the shape of the dissociation curve. These observations are relevant to the interpretation of saturation and partial pressure measurements and will be discussed in relation to higher and lower levels of arterial oxygenation.

AT HIGHER LEVELS OF OXYGENATION $\left(\mathrm{PaO}_{2}>12 \mathrm{kPa}\right.$, $\mathrm{SaO}_{2}>97 \%$ ).

As long as oxidative insults to the developing retina are thought to contribute to the pathogenesis of the retinopathy of prematurity, excessively high partial pressures of oxygen in arterial blood during oxygen treatment must be avoided. There is no need to consider here exactly what constitutes 'excessive', but for the sake of argument let us say that the partial pressure should not be allowed to exceed 12 $\mathrm{kPa}$. Given fetal haemoglobin and a normal $\mathrm{pH}$, this corresponds to a saturation of about $97 \%$, which is well on to the flat part of the dissociation curve. It is easy to show that even if the limits of accuracy of an oximeter were as good as $\pm 2 \%$ a partial pressure of $18 \mathrm{kPa}$ is compatible with a saturation reading of $97 \%$. If the oximeter sometimes underestimated the true saturation by as much as $5 \%$ it would be necessary to keep the reading below $92 \%$ in order to be sure of not exceeding a partial pressure of $12 \mathrm{kPa}$.

Clearly, oximetry alone cannot guard against hyperoxia on the upper reaches of the dissociation curve and, in the case of a preterm baby receiving oxygen, partial pressure should be monitored when the saturation is greater than $90 \%$. Preterm babies with good lungs will of course exceed $90 \%$ saturation when breathing air, but in these circumstances concern about excessive partial pressures is rarely, if ever, relevant. In cases where hyperoxia is not a concern oximetry provides a satisfactory and reliable means of monitoring oxygen treatment and a saturation in the range of $90-95 \%$ should be ideal.

AT LOWER LEVELS OF OXYGENATION $\left(\mathrm{PaO}_{2} 6-10 \mathrm{kPa}\right.$, $\mathrm{SaO}_{2}$ 85-95\%).

During neonatal intensive care the partial pressure of oxygen is often maintained at levels that fall on the steep part of the dissociation curve. This is mainly influenced by the spectre of retinopathy, although sometimes lung function is too poor for arterial oxygenation to be improved beyond this point. But, even when it is possible to improve oxygenation, there may be an argument for not attempting to improve it much beyond the minimum required for tissue oxygenation as this usually means using a higher mean airway pressure, which probably increases the risk of barotrauma and chronic lung disease.

The steep part of the dissociation curve is a dangerous place, though, where quite small changes 
in oxygen tension produce large changes in saturation, and a change in $\mathrm{pH}$ has a much greater effect on saturation than it would on the upper reaches of the curve. For these reasons arterial oxygenation at lower levels is more sensitively monitored by an oximeter than by an oxygen electrode. As an example, take a change in arterial $\mathrm{pH}$ from $7 \cdot 4$ to 7.25 at a constant $\mathrm{PaO}_{2}$ of $6 \mathrm{kPa}$. The saturation will fall from about $88 \%$, which is satisfactory, to about $80 \%$, which may be too low for adequate tissue oxygenation at a $\mathrm{PaO}_{2}$ of $6 \mathrm{kPa}$. An oximeter will detect this, a device measuring partial pressure will not.

As a guide saturations above $85 \%$ are satisfactory, between $85 \%$ and $80 \%$ may be satisfactory if the partial pressure is above $6 \mathrm{kPa}$, and below $80 \%$ are unsatisfactory: that is, assuming a normal cardiac output and haemoglobin concentration in each case.

Despite the benefits of monitoring saturation at lower levels of oxygenation partial pressure still cannot be ignored because it determines the rate and duration of oxygen transfer to the tissues and also the muscle tone in the wall of the ductus arteriosus and the pulmonary arterioles. ${ }^{4}$ These effects cannot be exactly quantified, but the $\mathrm{PaO}_{2}$ should probably be kept above $5 \mathrm{kPa}$ whatever the saturation.

\section{Pulse oximetry in practice}

The pulse oximeter is an easy instrument to use. It needs neither calibration nor a warm up period and so gives usable data from the moment it is fixed to the patient. The response time is fast, and the correlation between oximeter readings and direct measurements of saturation on arterial blood is good. The mean absolute difference between oximeter readings and direct measurements of saturation is in the region of $3 \% .{ }^{5}$ The display of heart rate could be an important feature if the oximeter is used during resuscitation in the labour ward, which might be a useful role for the instrument. The probes supplied with the current oximeters are adequate rather than ideal, and could undoubtedly be improved with babies in mind. Compared to the problems of skin damage from transcutaneous electrodes, however, there is little to complain about. Oximeters are upset by a lot of patient activity and will alarm frequently if the limits are not set wide in these circumstances. Reliable readings can be expected only during quiet periods. The state of peripheral perfusion seems less critical to oximeters than to transcutaneous monitors, and they are rather less influenced by oedema. Jaundice has a slight effect on oximeter readings, but too little to matter with modern instruments. The oximeter probe must be shielded from phototherapy lights or it will not function properly.

\section{Conclusion}

It is tempting to regard oximeters mainly as rivals to transcutaneous oxygen monitors and to debate the relative merits of the two systems under similar circumstances. It is true that oximetry is useful for routine non-invasive monitoring of oxygen treatment on many occasions, but to limit its use to that purpose is to underestimate the potential value of measuring saturation in addition to partial pressure during intensive care, particularly at lower levels of oxygenation. Barriers to the acceptance of oximetry in neonatology should be easily overcome once it is seen as an additional means of monitoring rather than an alternative.

\section{References}

${ }^{1}$ Hellegers AE, Schruefer JJP. Nomograms and empirical equations relating oxygen tension, percentage saturation and $\mathrm{pH}$ in maternal and fetal blood. Am J Obstet Gynecol 1961;81:377-83.

2 Wasunna A, Whitelaw AGL. Pulse oximetry in preterm infants. Arch Dis Child 1987;62:957-8.

${ }^{3}$ Southall DP, Bignall S, Stebbens VA, Alexander JR, Rivers RPA, Lissauer T. Pulse oximeter and transcutaneous PO2 measurements in neonatal and paediatric intensive care. Arch Dis Child 1987;62:882-8.

4 Huage A. Hypoxia and pulmonary vascular resistance. The relative effects of pulmonary arterial and alveolar PO2. Acta Physiol Scand 1969;76:121-30.

${ }^{5}$ Fanconi S, Doherty P, Edmonds JF, Barker GA, Bohn DJ. Pulse oximetry in paediatric intensive care: comparison with measured saturations and transcutaneous oxygen tension. J Pediatr 1985;107:362-6.

\section{P R F DEAR}

Department of Paediatrics and Child Health, St James's University Hospital, Leeds

(See pages $882-8$ and 957-8.) 